\title{
Critical value of symmetry breaking parameter in the phase transition of decay rate
}

\author{
Hungsoo Kim ${ }^{a}$, Soo-Young Lee ${ }^{b}$, Sahng-Kyoon Yoo $^{c}$, D.K.Park ${ }^{b}$, Jae Kwan Kim ${ }^{a}$ \\ a Department of Physics, Korea Advanced Institute of Science and Technology, \\ Taejon, 305-701, Korea. \\ ${ }^{b}$ Department of Physics, Kyungnam University, Masan, 631-701, Korea. \\ ${ }^{c}$ Department of Physics, Seonam University, Namwon, Chunbuk, 590-711, Korea.
}

(July 3, 2018)

\begin{abstract}
Phase transition of decay rate from quantum tunneling to thermal activity regimes is investigated in $(3+1)$-dimensional field theories with symmetry breaking term $f \phi$. By applying the two independent criteria for the sharp first-order transition to the same model, the upper and lower bounds of critical value of the symmetry breaking parameter are obtained. Unlike two dimensional case continuum states of the fluctuation operator near sphaleron solution play an important role to determine the type of transition.
\end{abstract}


Recently, much attention is paid for the phase transition of the decay rate from thermal activity to quantum tunneling regimes in $(1+\mathrm{d})$-dimensional field theories when $d=1[1]$, $d=2[2]$, and $d=3[3]$. Especially, $d=3$ case, which is a concern of the present letter, has an abundant applications in cosmology[4] and particle physics[5,6]. In this problem potential is usually choosed, for simplicity, as an asymmetric double well potential

$$
V_{1}[\phi]=\frac{1}{2}\left(\phi^{2}-1\right)^{2}-f \phi,
$$

where $f$ is symmetry breaking parameter whose range is $0<f<4 / 3 \sqrt{3}$. In fact, Eq.(1) is a tree level potential of the temperature-dependent corrections $[7,8]$. The analysis of the phase transition for the temperature-dependent effective potential by the method used in this letter is expected to be very complicated and will be discussed elsewhere. After taking an appropriate scaling transformation it is easy to show that the system with potential (1) exhibits a same physics with a system whose potential is[9]

$$
V_{2}[\phi]=\frac{1}{4} \phi^{4}-\phi^{3}+\frac{1}{2} \delta \phi^{2},
$$

if $\delta$ has a relation with $f$ as follows:

$$
f=\frac{2(2-\delta)}{(3-\delta)^{\frac{3}{2}}} .
$$

From the range of $f$ one can see that the parameter $\delta$ can vary between 0 and 2. In the limit $\delta \rightarrow 2$ (or $f \rightarrow 0$ ), we can use the usual thin wall approximation method[10]. In the opposite limit $\delta \rightarrow 0$ (or $f \rightarrow 4 / 3 \sqrt{3}$ ), the height of the barrier between the two vacua vanishes. Since the radius of bubble is smaller than the wall thickness, this limit is frequently called thick wall limit[4].

In Ref.[3] Ferrera has shown that the type of the phase transition of decay rate is sharp first-order when $f=0.25$ and $f=0.55$, and smooth second-order when $f=0.75$ by solving the equation of motion numerically with an aid of the multigrid method. This means that there exists a critical value $f_{*}$ (or $\delta_{*}$ ), which distinguishes the type of transition. The multigrid method, however, is not useful to determine $f_{*}\left(\right.$ or $\left.\delta_{*}\right)$ because it is impossible to treat temperature continuously due to the fixed grids. Furthermore, it may be highly difficult to solve the equation of motion numerically in realistic models. It is, therefore, important to determine $f_{*}$ without solving equation of motion. It may be worthwhile noting that the 
terminology "phase transition" used in the present letter does not mean the generic transition of the physical system's phase but the transition between the two different instanton regimes.

In this letter we will evaluate the lower and upper bounds of $f_{*}\left(\right.$ or $\left.\delta_{*}\right)$ by using two independent criteria for the sharp transition.

Let us start with Euclidean action

$$
S_{E}[\phi]=\int d^{4} x\left[\frac{1}{2}\left(\frac{\partial \phi}{\partial \tau}\right)^{2}+(\vec{\nabla} \phi)^{2}+V_{2}[\phi]\right],
$$

which yields an equation of motion

$$
\frac{\partial^{2} \phi}{\partial \tau^{2}}+\vec{\nabla}^{2} \phi=V_{2}^{\prime}[\phi]
$$

Since zero temperature bounce solution $\phi_{B}$ has $O(4)$ symmetry[11], the equation of motion for $\phi_{B}$ is reduced to

$$
\frac{d^{2} \phi_{B}}{d R^{2}}+\frac{3}{R} \frac{d \phi_{B}}{d R}=V_{2}^{\prime}\left[\phi_{B}\right]
$$

where $R=\sqrt{\tau^{2}+\vec{x}^{2}}$. Thus, the classical action for $O(4)$ symmetric solution is

$$
S_{4}=2 \pi^{2} \int_{0}^{\infty} d R R^{3}\left[\frac{1}{2}\left(\frac{d \phi_{B}}{d R}\right)^{2}+V_{2}\left[\phi_{B}\right]\right] .
$$

At high temperature thermal activity is governed by static sphaleron solution[12] which has a $O(3)$ symmetry. Hence, the equation of motion for the sphaleron solution $\phi_{s p h}$ is

$$
\frac{d^{2} \phi_{s p h}}{d r^{2}}+\frac{2}{r} \frac{d \phi_{s p h}}{d r}=V_{2}^{\prime}\left[\phi_{s p h}\right]
$$

where $r=\sqrt{\vec{x}^{2}}$, and, thus, the classical action for this configuration is

$$
S_{s p h}=\tau_{s p h} S_{3}
$$

Here, $\tau_{s p h}$ is the period of small fluctuation around $\phi_{s p h}$ and

$$
S_{3}=4 \pi \int_{0}^{\infty} r^{2}\left[\frac{1}{2}\left(\frac{d \phi_{s p h}}{d r}\right)^{2}+V_{2}\left[\phi_{s p h}\right] .\right.
$$

At a finite temperature $T$ the solution with period $1 / T$ has an information on the decay rate[4, 13], which is a result of the saddle point approximation. 
The possible type of transition of decay rate from thermal activity to quantum tunneling regimes is thoroughly discussed in Ref.[14] within quantum mechanical models. By using a relation

$$
\frac{d S_{E}}{d \tau}=E
$$

where $\tau$ and $E$ are the period of solution and energy, respectively, Ref.[14] has shown that the Euclidean action for the finite temperature solution meets $S_{3} / T$-curve smoothly at $T_{s p h}=1 / \tau_{s p h}$. The general features of action-vs-temperature for the first- and secondorder transitions are shown at Fig.1. Although it is not general criterion for the sharp transition, one can see from Fig.1(b) that $S_{s p h}$ is larger than $S_{4}$ for the comparatively strong first-order transition.

In spite of quantum mechanical ground of Ref.[14] its generalization to field theories is straightforward since Eq.(10) holds even in field theories as a saddle point equation[15]. So, we can use a condition $S_{s p h}>S_{4}$ for a strong first-order transition. Since $S_{3}$ and $S_{4}$ have been obtained in Ref.[9] numerically

$$
\begin{aligned}
& S_{3}=\frac{64 \pi}{81}(2-\delta)^{-2} \sqrt{\delta}\left(\beta_{1} \delta+\beta_{2} \delta^{2}+\beta_{3} \delta^{3}\right), \\
& S_{4}=\frac{4 \pi^{2}}{3}(2-\delta)^{-3}\left(\alpha_{1} \delta+\alpha_{2} \delta^{2}+\alpha_{3} \delta^{3}\right),
\end{aligned}
$$

where $\beta_{1}=8.2938, \beta_{2}=-5.5330, \beta_{3}=0.8180, \alpha_{1}=13.832, \alpha_{2}=-10.819$, and $\alpha_{3}=$ 2.0765, the remaining one we have to calculate is $\tau_{s p h}$.

To obtain $\tau_{s p h}$ let us consider the small thermal fluctuation around the sphaleron:

$$
\phi(\tau, \vec{r})=\phi_{s p h}(r)+\eta(\tau, \vec{r}) .
$$

Inserting it to the field equation, one can show directly that $\eta(\tau, \vec{r})$ obeys

$$
\hat{l} \eta=\hat{h} \eta+\hat{G}_{2}[\eta]+\hat{G}_{3}[\eta],
$$

where

$$
\begin{aligned}
\hat{l} & =\frac{\partial^{2}}{\partial \tau^{2}} \\
\hat{h} & =-\vec{\nabla}^{2}+\left(3 \phi_{s p h}^{2}-6 \phi_{s p h}+\delta\right), \\
\hat{G}_{2}[\eta] & =3\left(\phi_{s p h}-1\right) \eta^{2}, \\
\hat{G}_{3}[\eta] & =\eta^{3} .
\end{aligned}
$$


After neglecting $\hat{G}_{2}[\eta]$ and $\hat{G}_{3}[\eta]$ which are higher order terms of $\eta$, one can show easily

$$
\tau_{s p h}=\frac{2 \pi}{\sqrt{\left|h_{0}\right|}}
$$

where $h_{0}$ is negative eigenvalue of $\hat{h}$ operator. Since eigenfunction for the negative eigenvalue is angle-independent, we have to solve the spectra in the radial equation

$$
\left[-\frac{d^{2}}{d r^{2}}-\frac{2}{r} \frac{d}{d r}+U(r)\right] u_{n}(r)=h_{n} u_{n}(r),
$$

where $U(r)=3\left(\phi_{s p h}^{2}-2 \phi_{s p h}\right)+\delta$. The eigenvalues for the negative mode and first positive mode are obtained numerically(See Fig.2). Fig.3 shows the numerical result of $S_{s p h} / S_{4}$ when Eq.(11) is used. In this case we get a condition $\delta>\delta_{1}^{A}=1.45$ for the strong first-order transition. Since, however, the author of Ref.[9] obtained $S_{3}$ and $S_{4}$ by fitting formula with only three parameters, Eq.(11) can be rough estimation. So, we calculated again $S_{3}$ and $S_{4}$ numerically by using the simple shooting method, which results in $\delta>\delta_{1}=1.42$, which is lying within his fitting error, as shown in Fig.3.

The opposite bound is obtained by using a criterion for the first order transition developed at Ref.[16]. In fact, authors of Ref.[16] obtained the condition for the sharp transition analytically which can be summarized as follows:

$$
<u_{0} \mid f\left[u_{0}\right]><0
$$

where

$$
\begin{aligned}
f\left[u_{0}\right]= & -\left.\frac{1}{2} \frac{\delta \hat{G}_{2}}{\delta \eta}\right|_{\eta=u_{0}}\left[\hat{h}^{-1}+\frac{1}{2}\left(\hat{h}+4 \omega_{s p h}^{2}\right)^{-1}\right] \hat{G}_{2}\left[u_{0}\right] \\
& +\frac{3}{4} \hat{G}_{3}\left[u_{0}\right]
\end{aligned}
$$

$<a \mid b>$ is usual inner product, and $u_{0}$ is negative mode of $\hat{h}$. Noting that the angle dependent eigenfunctions do not contribute to $<u_{0} \mid f\left[u_{0}\right]>$ and using explicit forms of $\hat{G}_{2}$ and $\hat{G}_{3}$ given at Eq.(14), one can reduce the condition (17) in our case to

$$
I_{1}+I_{2}<0
$$

where 


$$
\begin{aligned}
& I_{1}=\frac{3}{4}<u_{0}\left|\hat{G}_{3}\left[u_{0}\right]>-\left(\frac{1}{h_{0}}+\frac{1}{2} \frac{1}{h_{0}+4 \omega_{s p h}^{2}}\right)\right|<u_{0}\left|\hat{G}_{2}\left[u_{0}\right]>\right|^{2}, \\
& I_{2}=-J_{1}-J_{2} .
\end{aligned}
$$

Here,

$$
\begin{aligned}
& J_{1}=\sum_{n \geq 1}\left(\frac{1}{h_{n}}+\frac{1}{2} \frac{1}{h_{n}+4 \omega_{s p h}^{2}}\right)\left|<u_{n}\right| \hat{G}_{2}\left[u_{0}\right]>\left.\right|^{2}, \\
& J_{2}=\int d k\left(\frac{1}{h_{k}}+\frac{1}{2} \frac{1}{h_{k}+4 \omega_{s p h}^{2}}\right)\left|<u_{k}\right| \hat{G}_{2}\left[u_{0}\right]>\left.\right|^{2} .
\end{aligned}
$$

$u_{0}, u_{n}(n=1,2, \cdots)$, and $u_{k}$ are negative mode, discrete positive modes, and continuum states in the radial equation (16), respectively, and $h_{0}, h_{n}$, and $h_{k}$ are corresponding eigenvalues. Since $I_{1}>0$ and $I_{2}<0$, the competition of $I_{1}$ and $I_{2}$ determines the type of transition. Numerical calculation shows that there exists only one positive discrete mode in the radial eigenvalue equation (16). Hence, $J_{1}$ is simply

$$
J_{1}=\left(\frac{1}{h_{1}}+\frac{1}{2} \frac{1}{h_{1}+4 \omega_{s p h}^{2}}\right)\left|<u_{1}\right| \hat{G}_{2}\left[u_{0}\right]>\left.\right|^{2} .
$$

Although the criterion for the sharp transition (19) is analytic expression, it is highly difficult to treat the continuum states numerically. In order to escape this difficulty we change $J_{2}$ to its upper bound $J_{2}^{M}$

$$
\begin{aligned}
J_{2} \rightarrow & J_{2}^{M} \\
= & \left(\frac{1}{\delta}+\frac{1}{2} \frac{1}{\delta+4 \omega_{s p h}^{2}}\right) \int d k\left|<u_{k}\right| \hat{G}_{2}\left[u_{0}\right]>\left.\right|^{2} \\
= & \left(\frac{1}{\delta}+\frac{1}{2} \frac{1}{\delta+4 \omega_{s p h}^{2}}\right) \\
& \times\left[<\hat{G}_{2}\left[u_{0}\right]\left|\hat{G}_{2}\left[u_{0}\right]>-\right|<u_{0}\left|\hat{G}_{2}\left[u_{0}\right]>\right|^{2}-\left|<u_{1}\right| \hat{G}_{2}\left[u_{0}\right]>\left.\right|^{2}\right] .
\end{aligned}
$$

In deriving the upper bound $J_{2}^{M}$, we used the fact that the continuum eigenvalue is $\delta+k^{2}$. Since $J_{2}^{M}>J_{2}, I_{1}+I_{2}^{M}>0$ is a sufficient condition for the second-order transition, where $I_{2}^{M}=-J_{1}-J_{2}^{M}$. Results of numerical calculation for $I_{1}-J_{1}$ and $I_{1}-J_{1}-J_{2}^{M}$ are given at Fig.4. Since $I_{1}-J_{1}$ is the contributions of only negative and discrete positive modes to $<u_{0} \mid f\left[u_{0}\right]>$, the fact that $I_{1}-J_{1}>0$ in the full range of $\delta$ implies the contribution of the continuum states are very important for the occurrence of the sharp first-order transition. This is new feature which does not arise at $(1+1)$-dimensional case, in which the contribution 
of the continuum states is negligible[16]. Hence, we get $\delta<\delta_{2}=1.0245$ for the second-order transition. Combining Fig.3 and Fig.4 one can conclude $1.0245<\delta_{*}<1.42$ or in terms of $f 0.584<f_{*}<0.703$. Fig.5 shows bubble nucleation at $\delta=1.18$ which is calculated by the multigrid method, which shows the existence of the wiggly solution in the intermediate range of temperature.

In this letter, we derived the lower and upper bounds of $f_{*}$ or $\delta_{*}$ by applying the two independent criteria $S_{s p h}>S_{4}$ and $I_{1}+I_{2}<0$ to the same system simultaneously. Although one can obtain more accurate value of $f_{*}$ by solving equation of motion numerically in the simple model, this might be impossible for more complicated models like electroweak theory and real cosmological models whose potential are generally dependent on temperature explicitly. In these cases our method presented in this letter would be useful to determine the type of transition and the critical values of some parameters involved in the models. 


\section{REFERENCES}

[1] B. I. Ivlev and V. I. Mel'nikov, Phys. Rev. B36, 6889 (1987).

[2] J. Garriga, Phys. Rev. D49, 5497 (1994).

[3] A. Ferrera, Phys. Rev. D52, 6717 (1995).

[4] A. Linde, Nucl. Phys. B216, 421 (1983).

[5] S. Habib, E. Mottola, and P. Tinyakov, Phys. Rev. D54, 7774 (1996).

[6] A. Kuznetsov and P. Tinyakov, Phys. Lett. B406, 76 (1997).

[7] M. E. Carrington, Phys. Rev. D45, 2933 (1992).

[8] M. Dine, R. G. Leigh, P. Huet, A. Linde, and D. Linde, Phys. Rev. D46, 550 (1992).

[9] F. C. Adams, Phys. Rev. D48, 2800 (1993).

[10] S. Coleman, Phys. Rev. D15, 2929 (1977).

[11] S. Coleman, V. Glaser, and A. Martin, Commun. Math. Phys. 58, 211 (1978).

[12] N. S. Manton, Phys. Rev. D28, 2019 (1983).

[13] I. Affleck, Phys. Rev. Lett. 46, 388 (1981).

[14] E. M. Chudnovsky, Phys. Rev. A46, 8011 (1992).

[15] S. Y. Khlebnikov, V. A. Rubakov, and P. Tinyakov, Nucl. Phys. B367, 334 (1991).

[16] D. A. Gorokhov and G. Blatter, Phys. Rev. B56, 3130 (1997). 


\section{FIGURES}

FIG. 1. (a) Second-order transition from the thermal to the quantum regimes. (b) First-order transition from the thermal to the quantum regimes.

FIG. 2. Negative $\left(h_{0}\right)$ and discrete positive $\left(h_{1}\right)$ eigenvalues of $\hat{h}$ operator.

FIG. 3. Plot of $S_{s p h} / S_{4}$ with respect to $\delta$. The solid line is calculated by using Eq.(11) and the data points are obtained by shooting method. In the range of $\delta$ corresponding to $S_{s p h} / S_{4}>1$ the transition of the decay rate becomes comparatively strong first-order.

FIG. 4. Plots of $I_{1}-J_{1}$ and $I_{1}-J_{1}-J_{2}^{M}$ with respect to $\delta$. The fact that $I_{1}-J_{1}>0$ in the full range of $\delta$ means that the continuum states of operator $\hat{h}$ play an important role for the determination of the type of transition. From the plot $I_{1}-J_{1}-J_{2}^{M}$ we can conjecture that the transition becomes smooth second-order when $\delta<\delta_{2}$.

FIG. 5. Bubble nucleation at $\delta=1.18$. (a) Periodic bubble solution at $T=0.763 T_{\text {sph }}$. (b) Periodic wiggly solution at $T=0.994 T_{s p h}$. (c) $O(3)$ symmetric sphaleron solution at high temperatures. 


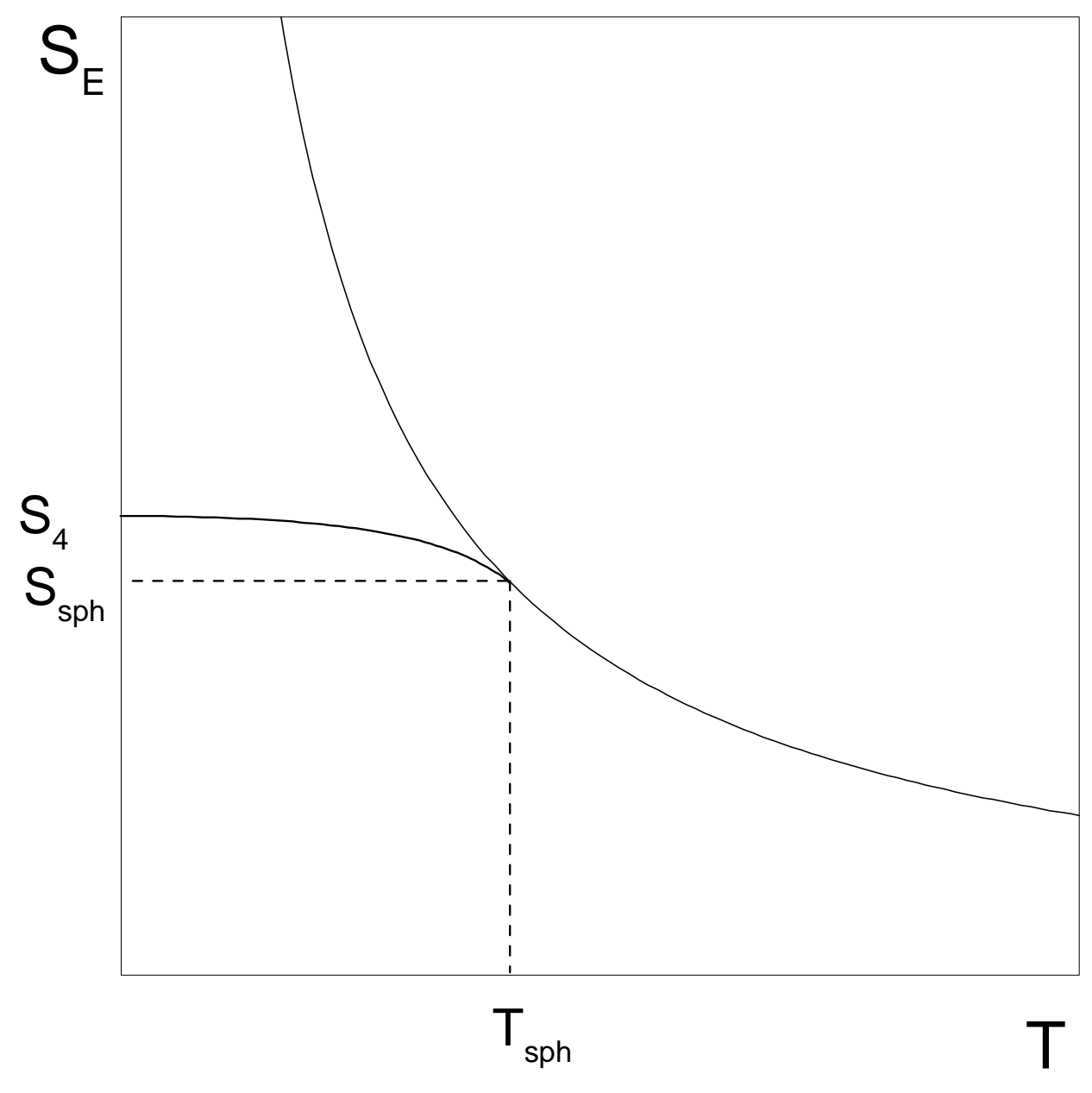

Fig.1 (a) 


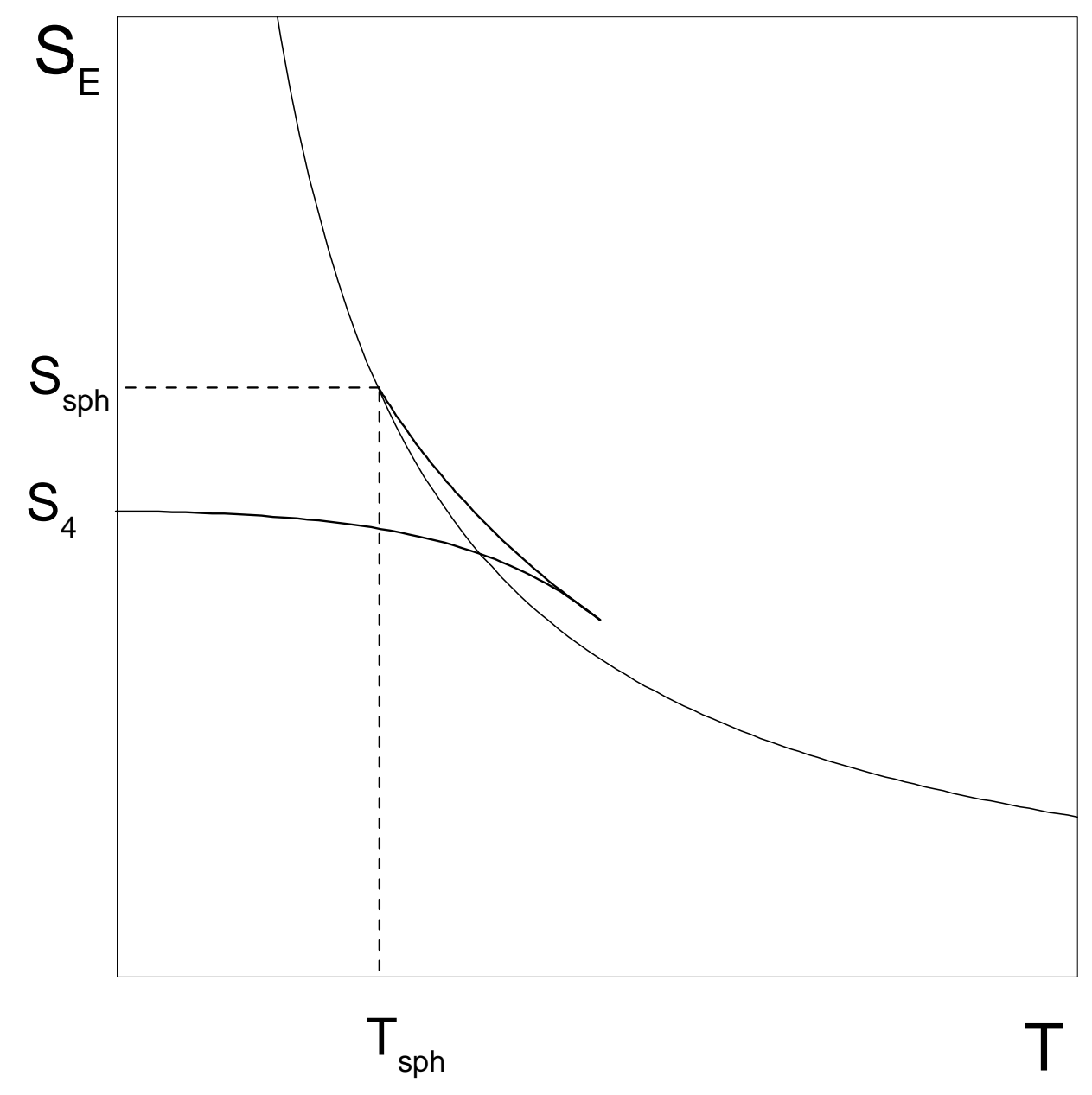

Fig.1 (b) 


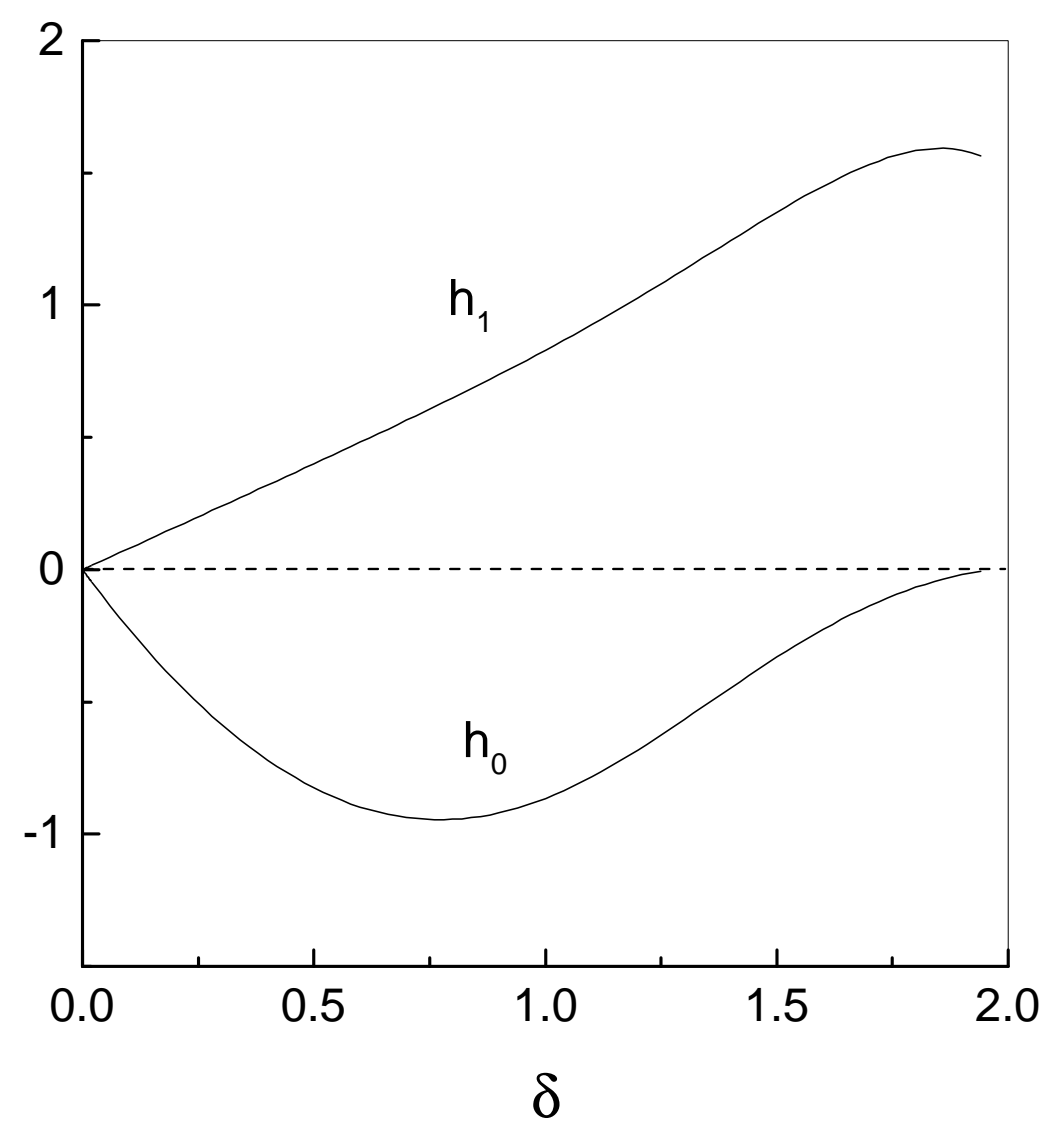

Fig.2 


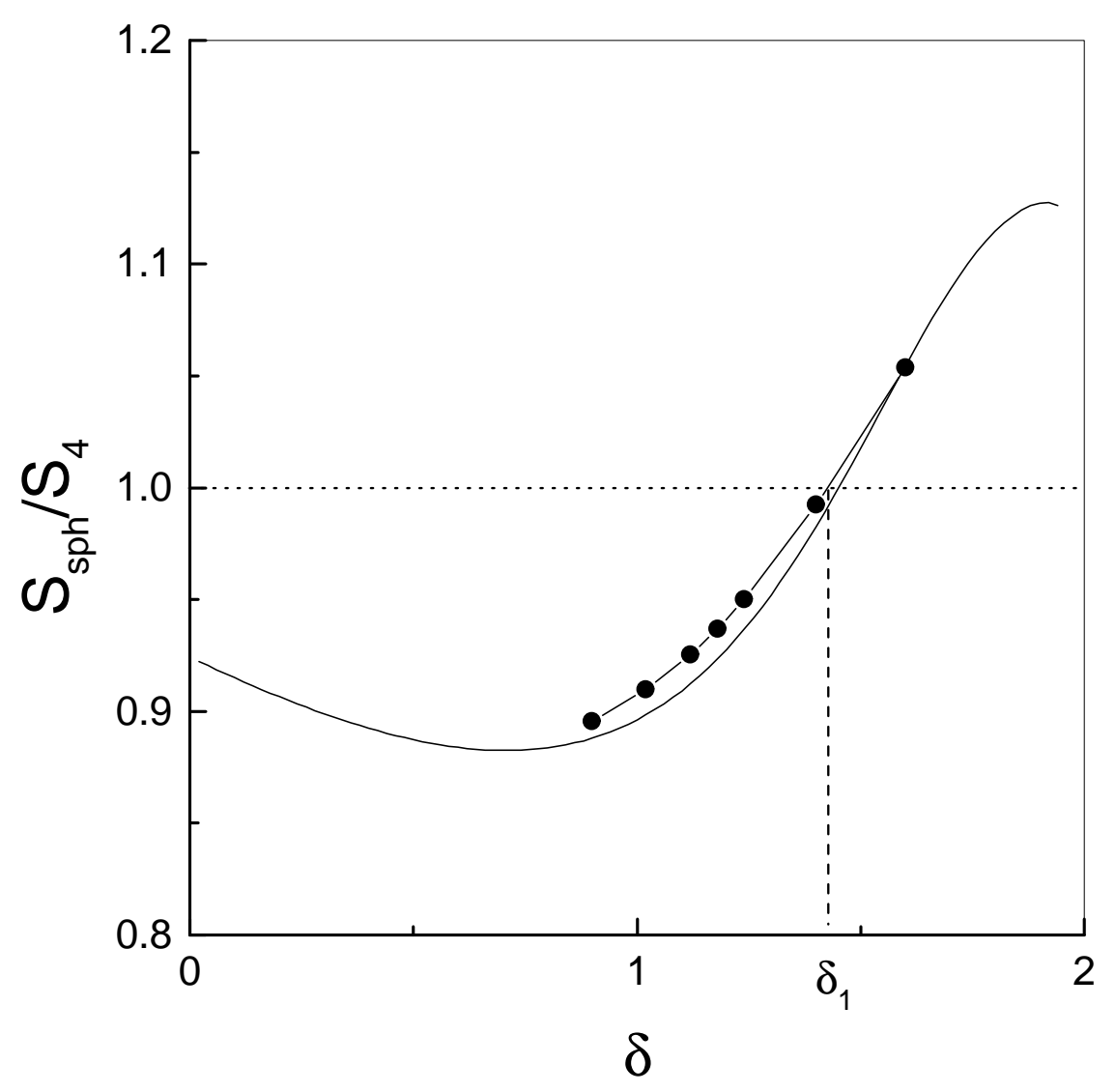

Fig.3 


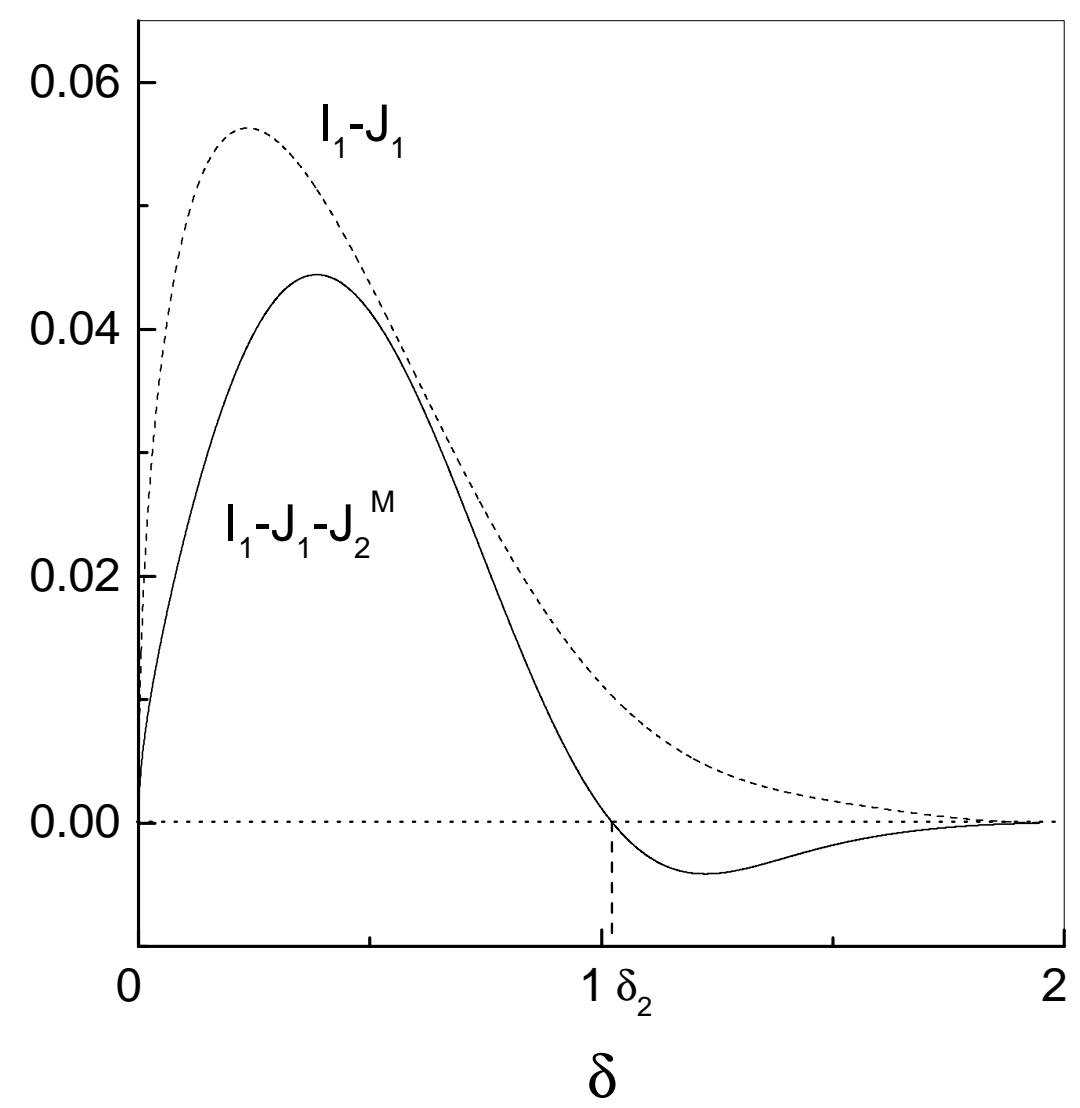

Fig.4 


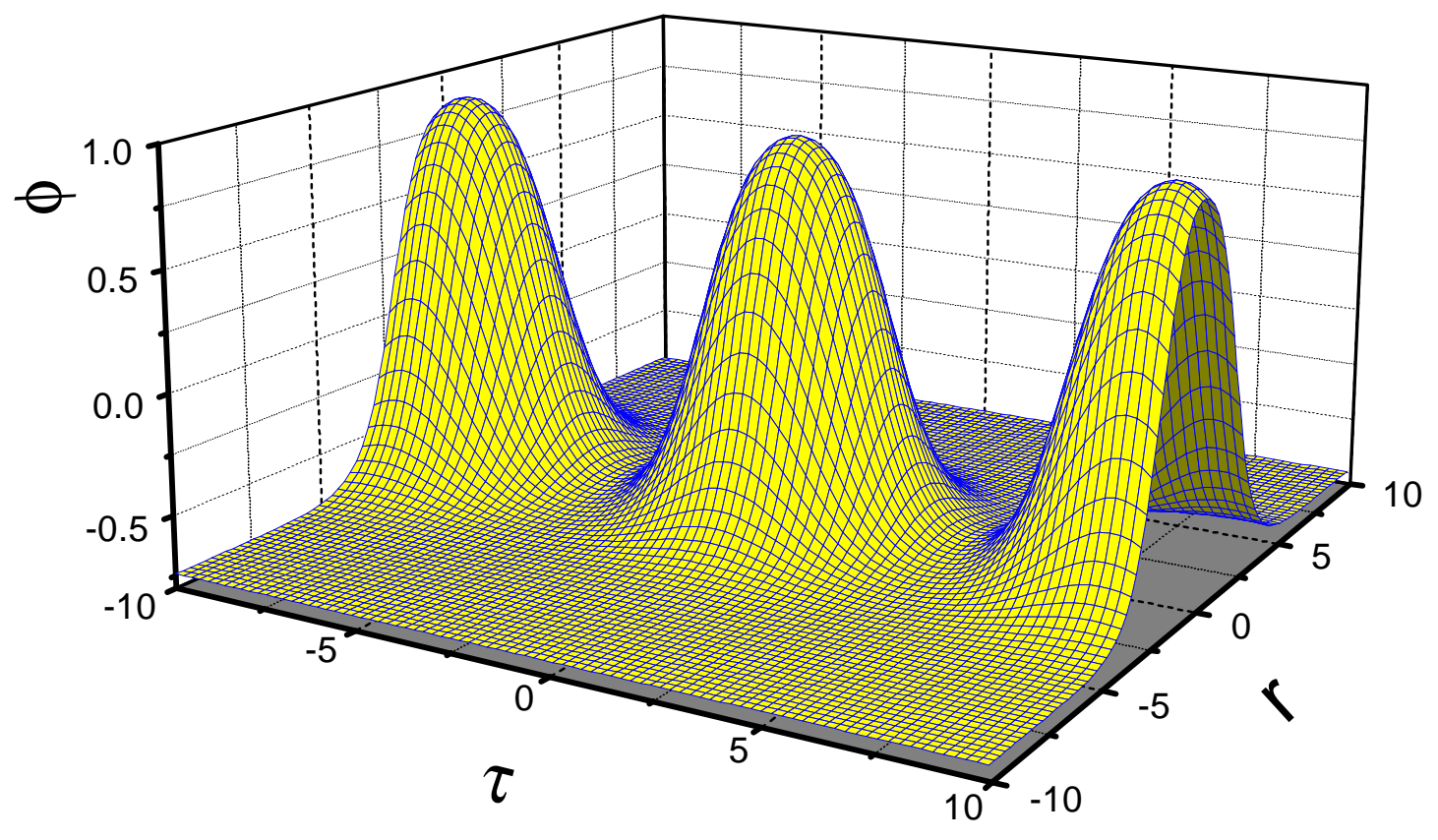

Fig.5 (a)T $=0.763 \mathrm{~T}_{\text {sph }}$ 


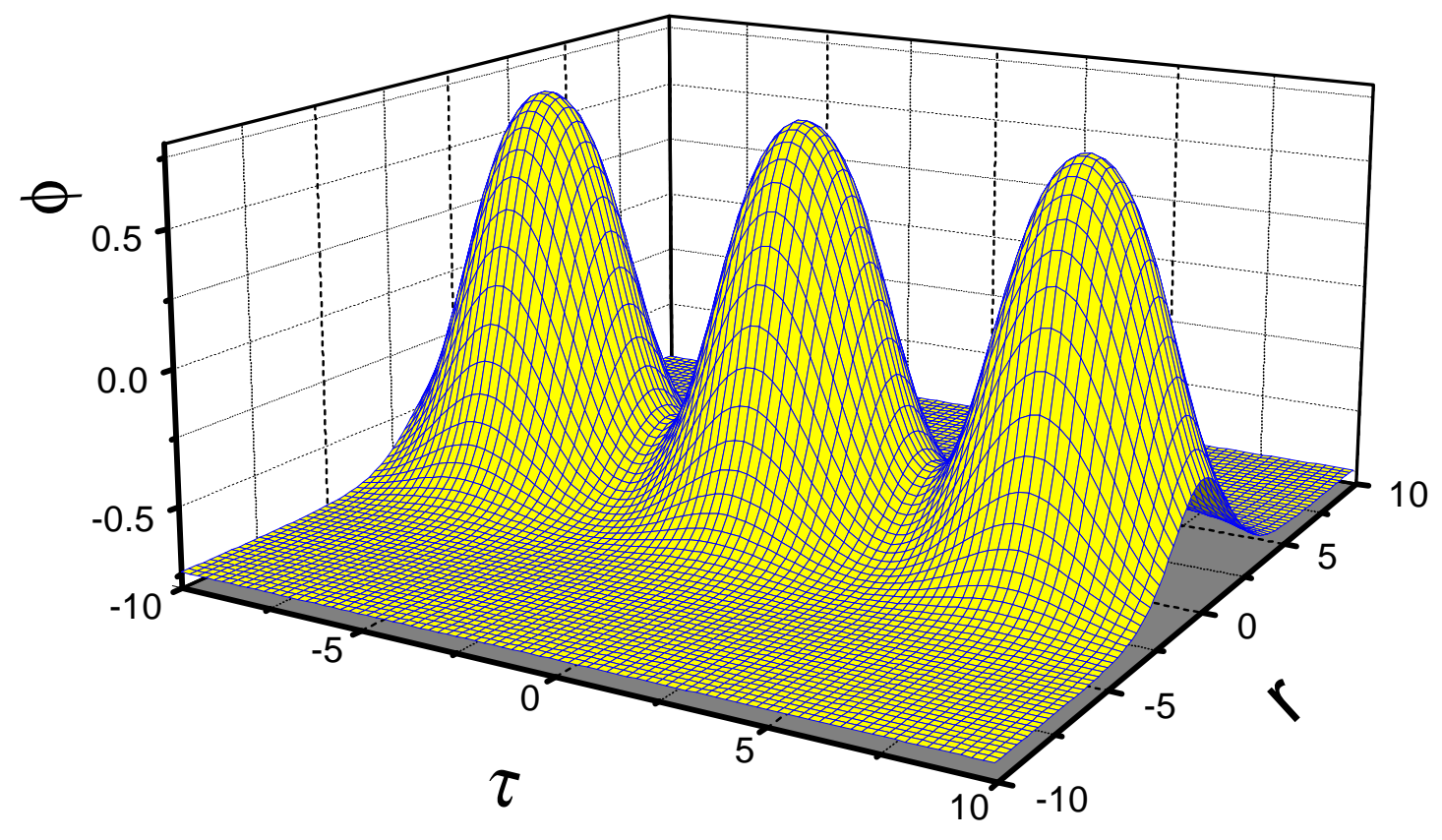

Fig.5 (b) $\mathrm{T}=0.994 \mathrm{~T}_{\text {sph }}$ 


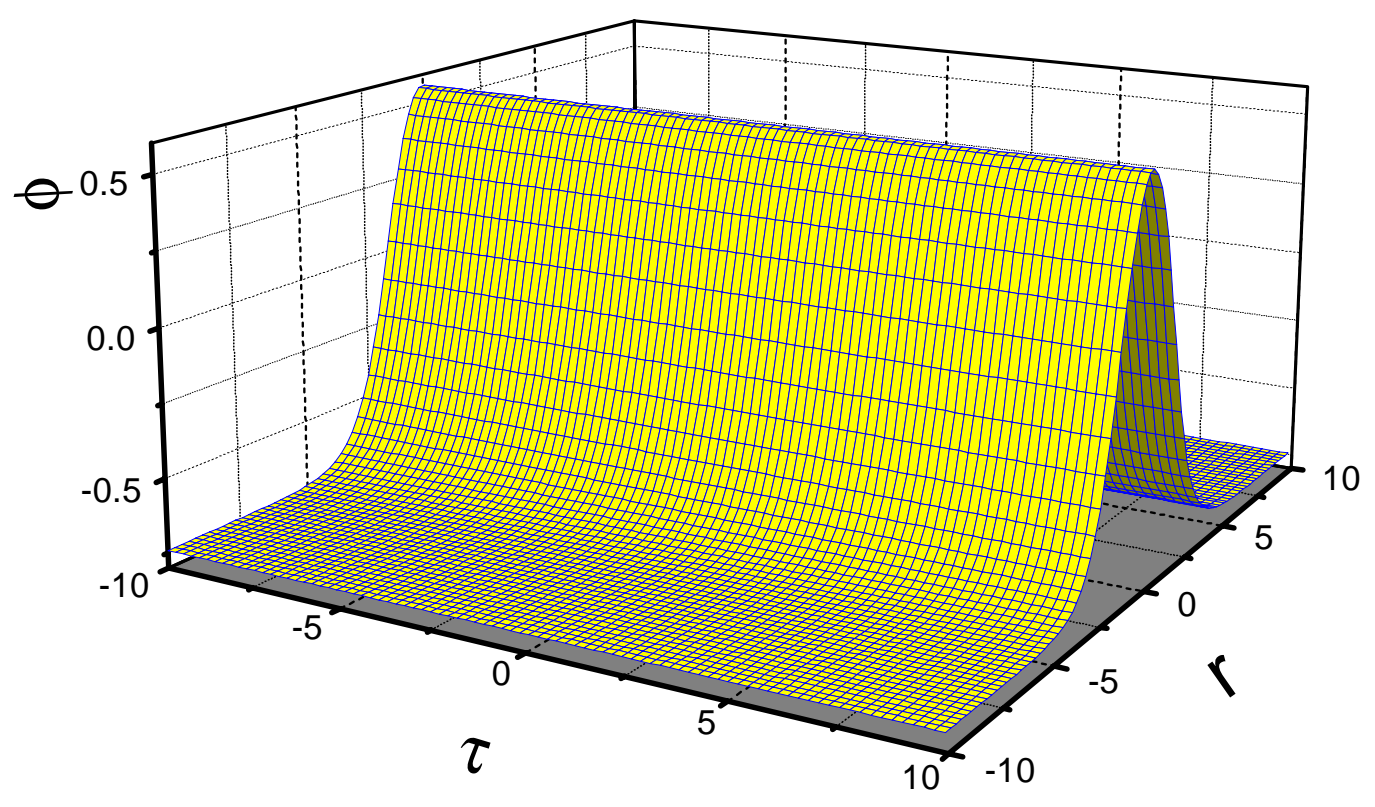

Fig.5 (c) 